our reported approach is also highly selective. Indeed, the determination of $10^{-11} \mathrm{M}$ of $\mathrm{Hg}$ (II) ions was not affected by the addition of $10^{-5} \mathrm{M}$ zinc, copper, lead or manganese or $10^{-7} \mathrm{M}$ silver. Furthermore, the introduction of $0.1 \mathrm{M}$ acetate, chloride, bromide or thiocyanate anions did not interfere with mercury analysis.

Such modified electrodes offer a simple, fast and reliable method for monitoring mercury in our environment. To the best of our knowledge, the developed method represents the lowest detection limit ever reported for $\mathrm{Hg}$ (II) using an electrochemical technique, which can be compared to the detection limit of expensive and relatively complicated methods ${ }^{3}$ such as inductively coupled plasma/mass spectrometry.

\section{Iva Turyan}

\section{Daniel Mandler}

Department of Inorganic and Analytical Chemistry,

The Hebrew University of Jerusalem, Jerusalem 91904, Israel

1. Bloom, N. S. \& Crecelius, E. A. Mar. Chem. 14, 49-59 (1983).

2. Szakacs, O., Lásztity, A. \& Horváth, Z. Analytica chim. Acta 121, 219-224 (1980).

3. Haraldsson, C., Westerlund, S. \& Öhman, P. Analytica chim. Acta 221. 77-84 (1989).

\section{Conduction by mantle hydrogen}

SIR - Karato's suggestion ${ }^{1}$ that hydrogen dissolved in olivine might contribute substantially to the electrical conductivity of the upper mantle has gained a sympathetic ear in the electromagnetic community. It has long been believed that the upper mantle at a depth of around $200 \mathrm{~km}$ has a relatively high electrical conductivity of about 0.1

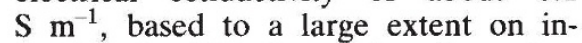
versions by Oldenburg of seafloor electrical data ${ }^{2}$, but this conductivity is one to two orders of magnitude larger than experimental measurements of dry, subsolidus olivine in the appropriate temperature range ${ }^{3}$. Although the requirement for high conductivities in the oceanic mantle has now been questioned on the grounds that coastlines distort the seafloor data ${ }^{4}$, partial melt, water and carbon have all been suggested at various times as conductivity enhancers for the upper mantle; now we have hydrogen.

Karato's idea, while excellent food for thought, is speculative. It is based on experimental evidence for large diffusivities of hydrogen in olivine ${ }^{5}$, but makes the assumption that the hydrogen exists as charged defects and extrapolates solubilities of 20-80 p.p.m. hydrogen to silicon (H:Si) at $300 \mathrm{MPa}$ to $100-1,000$ p.p.m H:Si at mantle pressures of about
$4 \mathrm{GPa}$ (130 km depth). While observations of hydrogen content in olivines from mantle xenoliths are only between 0 and 80 p.p. m $^{6}$, the large diffusivities of hydrogen imply that any higher concentrations of hydrogen would be lost during eruption.

Crystallographic anisotropy of diffusivity has been largely ignored by proponents of the hydrogen conduction hypothesis. Mackwell and Kohlstedt ${ }^{5}$ report diffusivities for all three crystallographic axes, with $D_{\mathrm{a}} \approx 10 D_{\mathrm{c}} \approx 100 D_{\mathrm{b}}$. Karato used only the $a$-axis diffusivities in his calculations, and while he acknowledged that the effect of anisotropy would reduce the conductivity of an isotropic mantle, this was not quantified. I have applied various mixing laws ${ }^{7}$ to predict the electrical conductivity of an isotropic mixture of all three axes, computing the parallel and series bounds of the conductivities along the three axes as implied by Karato's work, as well as the much more realistic geometric mean and effective medium average (an average of the series and parallel bounds, and shown in the figure). Also shown in the figure are results for the SO2 model of isotropic mantle conductivity based on laboratory measurements on olivine ${ }^{3}$. One sees that for 100 p.p.m hydrogen, the average conductivity of an isotropic mantle would be dominated by the conventional model of conductivity above $1,000{ }^{\circ} \mathrm{C} ; 1,000$ p.p.m. hydrogen produces conductivities which are only 5-6 times greater than the 'dry' model at $1,300{ }^{\circ} \mathrm{C}$. To achieve inferred mantle conductivities using the hydrogen model, one needs about $8,000-10,000$ p.p.m. $\mathrm{H}: \mathrm{Si}$. This might not be impossible; at 3 GPa hydrogen solubility in olivine could be as high as 5,000 p.p.m. ${ }^{8}$, based on extrapolation of the observed dependence of hydrogen solubility on water fugacity, hydrogen fugacity and oxygen fugacity at 50-300 MPa. However, water is likely to partition strongly into hydrous phases, or pyroxene, which contains about an order of magnitude more water than olivine ${ }^{6}$.

For the mantle deeper than about 200 $\mathrm{km}$, the isotropic model is appropriate. Deformation texture is likely to develop to some extent at shallower depths in the mantle and, as seismic observations suggest a preference for $a$-axis alignment in olivine, the axis of fastest hydrogen diffusion, this would be expected to produce an anisotropy in the bulk electrical properties if hydrogen did indeed dominate conduction. However, the responses of seafloor electrical experiments either show little anisotropy or show only that expected from the effect of coastlines. A complicating factor is that in the cooler, uppermost mantle electrical properties will no longer be dominated by silicates, be-

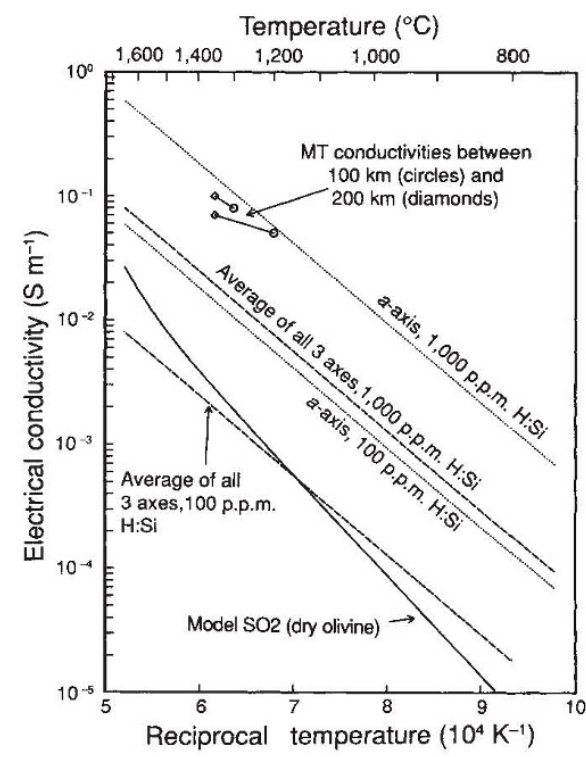

Conduction by hydrogen defects suggested in ref. 1 for 100 and 1,000 p.p.m. H:Si, based on diffusivities along the a-axis ${ }^{5}$ (dotted lines). Diffusivities along the $c$ - and $b$-axes are 10 and 100 times lower, respectively, and have been taken into account by computing averages of inferred conductivities along the three axes (broken lines). Model SO2 is based on laboratory measurements of dry, subsolidus olivine ${ }^{3}$ with conduction inferred to be by means of polaron hopping below $1,300{ }^{\circ} \mathrm{C}$ and magnesium vacancies above (solid line). Mantle conductivities are taken from conventional interpretation of seafloor magnetotelluric (MT) data which neglects the effect of coastlines $^{2,4}$. Inclusion of coastlines make the MT data compatible with the SO2 model of mantle conductivity ${ }^{4}$.

cause the activation energy for conduction by hydrogen $(1.3 \mathrm{eV})$ is similar to that in dry olivine $(1.6 \mathrm{eV})$, which has an electrical conductivity less than $10^{-5} \mathrm{~S} \mathrm{~m}^{-1}$ below $700{ }^{\circ} \mathrm{C}$.

Although electrical conduction in mantle olivine dominated by hydrogen has not been disproved, the large concentrations required to enhance bulk conductivity sufficiently, a probable low partition coefficient for water in olivine, and electrical models not requiring high mantle conductivities, conspire to make it less likely than previously supposed.

\section{Steven Constable}

Institute of Geophysics and Planetary Physics,

University of California, San Diego, La Jolla, California 92093-0225, USA

1. Karato, S. Nature 347, 272-273 (1990).

2. Oldenburg, D. W. Geophys. J. R. astr. Soc. 65, 359-394 (1981)

3. Constable, S. C., Shankland, T. J. \& Duba, A. J. geophys. Res. 97, 3397-3404 (1992).

4. Heinson, G. \& Constable, S. C. Geophys. J. Int. 110 159-179 (1992).

5. Mackwell, S. J. \& Kohistedt, D. L. J. geophys. Res. 95 5079-5088 (1990).

6. Bell, D. R. \& Rossman, G. R. Science 255, 1391-1397 (1992).

7. Shankland, T. J. \& Duba, A. G. Geophys. J. Int. 103 25-31 (1990).

8. Bai, Q. \& Kohlstedt, D. L. Nature 357, 672-674 (1992). 